\title{
Reflexiones acerca de la psicoterapia, la formación y el movimiento de la integración ${ }^{(1)}$
}

RESUMEN: Uno de los esfuerzos posiblemente más originales y novedosos en el campo de la psicoterapia viene dado en los últimos años por el discurso de la integración que, sin embargo, no parece haber contribuído, al menos en nuestro país, a concitar esfuerzos específicos entre los psicoterapeutas formados en diferentes líneas teórico/técnicas. La falta de debate, el formalmente respetuoso orillamiento del contenido de lo planteado, puede contribuir a sostener problemas y carencias existentes que es urgente abordar para contribuir a desarrollar adecuadamente la psicoterapia, especialmente en el Sistema Nacional de Salud: La formación (las diferentes escuelas y sus ritos), la acreditación oficial, el hostigamiento de su práctica en el Sistema Nacional de Salud, entre otros. Con la finalidad de contribuir a un necesario debate se presenta este trabajo.

PALABRAS CLAVE: Psicoterapia, debate, integración, formación
SUMMARY: One of the most original efforts in Psychotherapy in the last few years has been the introduction of the integration movement. Nevertheless it seems it has not generate in our country specific efforts among the psychotherapists trained in different theories and techniques. The lack of debate can contribute to mantain problems and shortages that already exist, and that ought to be solved to help to develop psychotherapy adequately, especially in the Public Health System: Training, the different theories and techniques, their ceremonies, the official acreditations, the difficulty in its practice in the health services, among others.

The aim of this article is to contribute to a necessary debate.

KEY WORDS: Psychotherapy, debate, integration, specialized training

Mucho se ha hablado y escrito acerca de la responsabilidad de los científicos en un mundo en el que las aplicaciones científicas han modificado las vidas de las gentes y en el que un ejercicio de la ciencia se realiza a través de los profesionales que, oficialmente cualificados, desarrollan y aplican conocimientos sobre la población. Así, no es de extrañar el aumento de la influencia social de la ciencia y sus disciplinas, así como de los profesionales, y junto a ello de la competencia entre diferentes ramas del saber aplicado con sus efectos de discusión, debate y crítica acerca de sus resultados, de su estatus, de su aplicación, etc.

(1) Texto elaborado a partir de la ponencia presentada en el Symposium Training in Psychotherapy for residents in Psychiatry and Clinical Psychology, presidido por el Dr. F. Montilla, en el Congreso Internacional de la Asociación Mundial de Psiquiatría (Madrid, Septiembre-Octubre 2001), organizado por la Asociación Mundial de Psiquiatría (AEN -SEP-SEPB) . 
Ciertamente, las aplicaciones de la ciencia no son posibles sólo por sí mismas o por el libre criterio del profesional. La responsabilidad de las aplicaciones dependen de la financiación de la investigación, de la comercialización del producto, de las organizaciones públicas que incorporan profesionales y prestaciones, de las líneas políticas impulsadoras, de las necesidades sentidas y expresadas por los ciudadanos, del uso (y abuso) de ciertos instrumentos científicos de rentabilidad comercial probada frente a otros, de las posiciones académicas y sus relaciones con la financiación externa, de las asociaciones científico-profesionales, etc.

De este modo la responsabilidad de la investigación/desarrollo de las aplicaciones científicas se configura en un entramado en red que conviene analizar en cada caso. En este sentido no hay que olvidar la responsabilidad de quienes forman "mentalidades" entre los científicos y profesionales. Mentalidades que llegan a pretender establecer legitimidad para el hostigamiento, (cuando no exterminio) de supuestos "enemigos" de aplicaciones científicas. Una de estas aplicaciones "hostigadas" es a mi juicio la psicoterapia. Sus hostigadores utilizan para ello artilugios elementales, como dificultar gravemente la posibilidad de su práctica en las instituciones públicas; o sofisticados, como anular las fuentes de financiación o construir mitos. Uno de los mitos más insistentes en torno a lo que nos ocupa es el de la debilidad científica de las psicoterapias como instrumento de comprensión y abordaje de los trastornos mentales, pretendiendo al tiempo hacer olvidar otras carencias en otras líneas de intervención, como las de la investigación de laboratorio químico-farmacéutico o la experimental.

Uno de los efectos de este hostigamiento me parece percibir que se traduce en el progresivo debilitamiento del debate abierto entre los psicoterapeutas en torno a diversas cuestiones de la psicoterapia (o psicoterapias, como vds. gusten). Los psicoterapeutas evitamos abordar (y tomar posiciones) en debates sobre asuntos que nos conciernen para así proteger (seguramente de manera equivocada) la dignidad de la psicoterapia y de su ejercicio. Pero así las ideas se hacen inanes, pues son las ideas interesantes las que incitan al debate, a la fructífera discusión civilizada.

Aún en esta situación, un estado de opinión creo que empieza a no ser minoritario y se refiere al estatus epistemológico de la psicoterapia o, si se quiere, al problema de coexistencia de diferentes paradigmas y su supuesta rápida solución mediante la apuesta por su inmediata integración.

Añadiré ahora que la discusión que inicio aspira más a ser civilizada que fructífera, pues sólo pretendo razonar alguna discrepancia.

Para empezar diré que creo que desde un punto de vista epistemológico, la actual coexistencia de diferentes paradigmas teórico-técnicos en psicoterapia, no supone el ejercicio de actividades contradictorias, sino precisamente epistémicamente complementarias. Quien objete 
que una cosa es la metodología o técnica de intervención y otra el pensamiento o teoría que lo sustenta, olvidará que en método científico toda técnica es también teoría y que en psicoterapia el anclaje teórico de la acción terapéutica es de una relevancia intensa para el psicoterapeuta, cuya acción no puede realizarse "a ciegas" de pensamiento.

Comparto la desconfianza ante la máxima que al parecer justifica y tiraniza alguna de las modalidades de concepción del proyecto de integración de algunos psicoterapeutas, según la cual el propósito de toda psicoterapia es la mejoría del paciente (así, si ése es el objetivo, "vale" la mezcla...). Una perogrullada que sólo deja de serlo si se añade que por "mejoría" es posible identificar diferentes cosas y que la psicoterapia puede e incluso debe hacer además otras cosas. En cualquier caso, cosas distintas a las que ofrece (y sólo parcialmente) la farmacología; distintas, es decir, de otra naturaleza. Y, naturalmente, esas cosas distintas están marcadas por el que-hacer teórico-técnico de cada paradigma en psicoterapia: Por ejemplo, los estímulos pueden ser ambientales o no, nuevos o no para el individuo, condicionados $\mathrm{o}$ incondicionados, discriminativos o no, etc., la personalidad constructivamente los significa (Pelechano,2000). En esta construcción de significado participan diferentes subsistemas: el biológico, el cognitivo, el afectivo-emocional, el inconsciente, el relacional...con una construcción singular a través de interacciones y acentuaciones se establecen conexiones entre ellos de manera que un afecto puede generar cogniciones y/o impulsar conductas; un cambio conductual puede modificar cogniciones y afectos; un cambio relacional puede generar cogniciones $\mathrm{y}$ conductas sobre nuevas emociones; o un cambio cognitivo puede generar a su vez afectos, conductas, y a veces relaciones, nuevos. Todo ello en interacciones singulares en que se podrá producir mayor o menos grado de conciencia en la organización e integración de la experiencia en un juego estratégico de relaciones comunicativas con el contexto del que participa. Se trata de múltiples principios organizadores de la construcción de significación. Significación que tiene consecuencias retroalimentadoras internas y también externas, relacionales, en el juego dinámico de los contextos de los que el individuo participa.

En estos principios organizadores, en su diversidad, en su distinta naturaleza, se basan los distintos paradigmas de la psicoterapia. De la mejor naturaleza de uno u otro de estos principios organizadores creo que ya no se discute.

En ciencia, el análisis de los elementos objetivos es central. Tan central que la metodología investigadora científica obliga a la diferenciación en el campo de la salud/enfermedad entre signos y síntomas (Pelechano en su último libro antes citado desarrolla -entre otras muchas- una impecable argumentación referida a las teorías de la personalidad que básicamente creo puedo tomar prestada para la psicoterapia). Los signos contienen los elementos ("hechos") objetivos, mientras que los síntomas contienen elementos ("hechos") 
DEBATES

que se relacionan con lo experiencial, interpretable. Los signos son indicadores objetivos, los síntomas se expresan a través de la transmisión del paciente de su padecer, sujeta por ende a elementos provenientes de lo relacional, cultural, simbólico, etc.

Las diferentes escuelas de psicoterapia comprometen definiciones radicalmente diferentes de signo y de síntoma: El psicoanálisis, la sistémica, la gestalt, el conductismo, el cognitivismo...defienden definiciones que poco o nada tienen que ver entre sí.

Pero eso no quiere decir que el conocimiento contrastado en psicoterapia o la metodología y/o la teoría en que cada línea se sustenta, hayan de ser consideradas negativamente desde un punto de vista científico o abandonadas por esas diferencias. Más bien me parece que la conclusión debe apuntar a que el conocimiento en la clínica de la psicoterapia contiene dificultades, problemas. Seguramente, en su reconocimiento, en la aceptación de la existente diversidad, en los diferentes "puntos de mira" de cada una de las líneas de estudio e intervención y en la capacidad de trabajar desde ellas en líneas abiertas hacia la intercomunicación y también hacia la contrastación de sus resultados (no sólo de "efectos", sino también de proceso o de estructura) y las bases que los sustentan, tal vez se encuentre una vía a recorrer que cortos, truncados caminos ha tenido hasta ahora. En este sentido la "integración", es un movimiento que creo necesario. Si por contra "integración" fuese el comienzo de una escuela psicoterapéutica más que suma elementos de diversas procedencias, es otra cosa.
Pero mis discrepancias no acaban ahí.

Dejaré de lado la afirmación tan larga y fuertemente sostenida durante los años 90 de que en otros países con investigación y criterio crítico mayor, la psicoterapia en sus diferentes líneas está de capa caída. Según mis noticias esto no ocurre en EEUU, Canadá, Australia, Reino Unido, Alemania o Italia, por ejemplo (el caso de Francia tal vez sea algo distinto, aunque posiblemente cada vez menos). Países todos ellos en los que, hasta donde alcanzo, las diferentes escuelas de psicoterapia gozan de una salud de hierro.

Al tiempo, acuciados por urgencias provenientes de otras lógicas -casi siempre económicas-, los investigadores y clínicos de la psicoterapia deglutimos la mentalidad de pragmatismo eclecticista como forma de adaptarnos a esas "urgencias" que de otros lados, no necesariamente ilegítimos, provienen, asentando así por otra vía fuera de la lógica del conocimiento, una injusta confusión entre lo que se ha venido a llamar "eclecticismo" e Integración. Pero así ¿no estaremos olvidando que "no hay nada más práctico que una buena teoría", como decía Bateson? Que una buena psicoterapia precisa para su ejecución y avance de la teoría y la técnica que le dan soporte y propician su desarrollo? ¿no será que para evitar abordar problemas como el del sectarismo, tan dominantes en el mundillo de la psicoterapia y sus ocultos, dañinos y rígidos modos de inter e intra-relación, buscamos orillarlos con propuestas de fórmulas "pragmáticas" para la psicoterapia, que dificultan aún más, si cabe, un 
DEBATES

acercamiento riguroso entre los diferentes esquemas referenciales?

Esta forma de autoindulgencia colectiva no me parece otra cosa que pereza, cuando no negligencia, intelectual. La confluencia de paradigmas debe desatarse desde la identificación de carencias y errores propios y su articulación con nuevas ideas, datos y hallazgos provenientes de la investigación. La coherencia teórico/técnica para ello resulta necesaria. Sin ésto, ¿no estaremos contribuyendo a generar una nueva escuela de psicoterapia, con sus didactas, con sus ritos...? Propugnar una mayoría de edad de la psicoterapia en base a la construcción de un paradigma "nuevo" desde este "caldillo" ¿no sería una salida escapista y por ello falsa?

Más relevante me parece otra cuestión: Que la psicoterapia carece epistemológicamente de estatuto propio. Y que, frente a ello, la integración es una solución. Dejaré de lado por ahora si realmente es una solución, además ¿carecer de estatuto es un defecto o es un rasgo de un conocimiento que se define por su carácter proteico, capaz de asimilar lo que proviene de tan variados lugares (investigación de procesos psicológicos básicos, en funcionamientos y dinámicas grupales, en los modos en que se produce, integra, transforma y transmite información relacional, etc., etc.)?

Me parece más exacto decir que gran parte de lo que produce la investigación más tarde o más temprano se incorpora y pasa a pertenecer a la psicoterapia (y éste puede ser uno de los elementos que nos ayuden a discriminar funcionamientos sectarios de escuelas psicoterapéuticas), que, desde su nacimiento se configura como territorio de aluvión de datos, ideas y métodos, que crea artefactos cuya asombrosa originalidad seguramente se encuentra en el hecho de constituírse en una verdadera enciclopedia que licúa o sintetiza saberes coetáneos sobre el hombre.

En este sentido si la psicoterapia no tiene estatuto epistemológico, al menos tiene un rasgo: es un saber de saberes. Su estigma así podría ser una virtud: la maleabilidad, a la que posiblemente se debe el hecho de las crisis de crecimiento habidas en su algo más de un siglo de historia. Crisis que han provocado alteraciones en los paradigmas dominantes, que se han traducido a su vez en cambios internos en cada uno de los paradigmas existentes, que se han ido "resolviendo" por la vía de la asimilación de conocimientos adyacentes, provenientes de otros paradigmas surgentes o de otras disciplinas: ¿Quién puede decir hoy que el conductismo es igual que hace 20 años? ¿El cognitivismo no ha modificado sustancialmente el modo de concebir y de operar de aquél? ¿Quién puede decir hoy que el psicoanálisis no se ha movido en 100 años con saberes provenientes por ejemplo de la teoría de la comunicación? ¿Quién puede negar que la teoría sistémica -que toma desde su origen tantos elementos de la cibernéticano tiene con el constructivismo un diálogo de amplio calado teórico/técnico? Pero ahora eso no es ya suficiente.

Tal vez sea preciso subrayar el carácter híbrido del saber psicoterapéutico, derivado de una peculiar y variable mezcla 
de elementos provenientes de la incorporación de materiales y procedimientos muy diversos. Pero este hecho del carácter híbrido no es una novedad y al tiempo no justifica ni avala por sí mismo modos de "integración" sumativos. Esos modos tampoco parecen responder o justificarse en evolución epistémica o de desarrollo de modelos teórico/técnicos, pero alcanzan una notoriedad que posiblemente desacredita los esfuerzos que se dirigen a intentar satisfacer la necesidad de introducir la norma del respeto intelectual y formal para acabar con las largas luchas fratricidas entre escuelas en función de predominancias de influencia, de poder (más o menos imaginario). Tampoco su notoriedad proviene de su entrada en temas o contenidos centrales en psicoterapia. Más parecen entenderse a la luz de la sociología del conocimiento: con la frecuente utilización de términos y discursos ideológicos generales poco definidos y sin duda bienintencionados sobre la integración y el multiculturalismo en un mundo global ¿qué psicoterapeuta de buena voluntad podría oponerse a algo tan "políticamente correcto" que se da en llamar "Integración" (como un cajón de sastre)? La sumatividad "integradora”, ¿no mostraría un respeto casi reverencial por todas las partes de modelos teórico/técnicos que incorpora? Creo por contra que aporta falta de respeto y de rigor. Y además que anula así el valor de la existencia de cierta incomodidad legítima y pertinente respecto a ese tipo de lucha por la dominancia entre modelos y al deseo de terminar con ello sin participar desde parte buscando introducir rigor, respeto, neutralidad, para un nuevo terreno de acercamiento, hacia una integración verdaderamente respetuosa, que atiende a las propuestas terapéuticas más que a los "popes" que las formulan ritualizadamente. Lo hasta aquí dicho no supone una desvalorización, sino una posición crítica: la difusión de algo en círculos más o menos relevantes de poder científico/profesional no otorga a ese algo más valor científico o utilidad profesional.

Por otra parte, quiero señalar lo que creo un riesgo: la integración en psicoterapia se dibuja de día en día como una "nueva" opción entre las demás. Que esta lucha anuncie un cambio de paradigma que pase o no por la integración, más allá de las buenas intenciones, dependerá a mi juicio del buen hacer de los psicoterapeutas en su clínica/investigación. De su buen-hacer, de su voluntad y su talento. De los buenos psicoterapeutas, quiero decir.

Porque tal vez ahí tenemos el problema, no en la falta de estatuto, en los distintos ejes vertebradores del conocimiento en psicoterapia, en los diferentes modelos en definitiva, sino en los psicoterapeutas, cuestión que luego he de retomar. Creo que la integración en psicoterapia, como movimiento que también puede ser, puede ya anotarse varios puntos: haber acentuado, subrayado desde la práctica, la necesidad del respeto mutuo (no igual a vivir de espaldas, con indiferencia mutua), la necesidad de acabar con las guerras fratricidas, con el sectarismo, la necesidad de mirar desde cada modelo los otros modos de mirar y hacer en psicoterapia y sus efectos. Y tal vez un punto especialmente: Ser un alegato contra las malas psicoterapias y contra los deficientes psicoterapeutas. 
Por otra parte, creo que no es triunfalismo decir que la calidad del ejercicio y la investigación en psicoterapia en los años 80 y 90, ha sellado un pacto con la ciudadanía en nuestro país: Ya no extraña a nadie que cualquier ciudadano, incluso de alta responsabilidad social, pueda acudir regularmente a un tratamiento psicoterapéutico. Las consecuencias positivas del hecho se han traducido en nuestro país entre otras, en que el vigente catálogo de prestaciones del Sistema Nacional de Salud incluya la psicoterapia, cuando se trataba de una práctica casi inexistente con anterioridad.

Pero tal vez ahora se están volviendo más evidentes algunas consecuencias negativas. Efectivamente, ¿no estaremos dando por buenos productos inmaduros o insuficientes?, ¿qué instrumentos de control de calidad de la prestación psicoterapéutica, de tan alta incidencia en la calidad de vida de un individuo, tenemos establecidos?, ¿cuáles son los sistemas de formación oficialmente acreditados garantizadores de la calidad? Sencillamente, no los tenemos.

La cuota de mercado a preservar que los modos de gestión de las aseguradoras vienen implantando en EEUU y algún país europeo ante el crecimiento, al parecer imparable, de la demanda de psicoterapia entre la población, puede llevar a olvidar que uno de los propósitos (explícitos o implícitos) de toda psicoterapia digna de tal nombre es la impugnación (que no el escamoteo) de la realidad vivida por nuestros pacientes. Hay un gesto de insubordinación contra lo establecido que late en la psicoterapia al postular la posibilidad de construir con nuestros pacientes una realidad distinta a la que traen. No cualquiera ni de cualquier forma: el rigor del ejercicio ha sido uno de los ejes organizadores de todo esquema referencial o línea teórico-técnica en psicoterapia, lo que ha podido llevar a alguna de sus escuelas a sesgos procedimentales deformantes, ritualizados sectariamente, para el acceso a la condición de psicoterapeuta, condición no recogida oficialmente de manera alguna.

Ante la búsqueda de esa "mayoría de edad" para la psicoterapia, tal vez sea el momento en que debamos pasar a establecer procedimientos que acrediten oficialmente el rigor suficiente en la formación de psicoterapeutas. Posiblemente en el campo de la psicoterapia, uno de los modos de romper con oscurantismos sectarios y sus "poderes" de escuelas y sacerdotes de sus verdades, puede estar en el establecimiento de formas regladas y supervisadas en el sistema público de formación de especialistas, en definitiva, en servicios sanitarios acreditados a tal fin. En este sentido, la propuesta en España de establecer un ACE (Área de Acreditación Específica) a desarrollar para esta formación en las especialidades de Psicología Clínica y Psiquiatría de acuerdo a un programa oficial específico a desarrollar en servicios sanitarios acreditados a tal fin, resulta a mi juicio del mayor interés. La necesaria transparencia en el ejercicio, la permeabilidad de los servicios, el intercambio abierto existente entre estos profesionales, la contrastada alta calidad de las prestaciones en los servicios públicos acreditados para la formación de especialistas, podrían además resultar un buen vehículo en el imprescin- 
dible proceso de contacto y contraste inter e intra modelos de psicoterapia.

El respeto por nuestros pacientes se expresa también en el compromiso del terapeuta con la terapia y en aportar un enfoque de adecuada potencia, capaz de aunar fuerzas de cambio desde una coherencia teórico-práctica abierta. Es nuestra responsabilidad. Tal vez, frente a los modos de hacer excluyentes en Psicoterapia, trabajar para establecer progresivamente procedimientos de garantía pública en la formación de psicoterapeutas y en la dirección de ofrecer pensamiento y técnica desde actitudes abiertas, favoreciendo sucesivos engarces posteriores en diferentes aspectos en psicoterapia, sea un camino que esté empezando a trazarse. Nuevos cauces para el nuevo objetivo que puede ser de todos: confluir. Es preciso discriminarlos de los oportunismos.

Son actitudes loables ya en marcha, que no han de negar el que cada uno de los niveles de análisis a los que remiten los diferentes enfoques psicoterapéuticos no son básicamente reducibles unos a otros, sino que implican aportaciones diversas a desarrollar, no a reducir o traducir porque las diferencias entre los niveles no permiten la traducción.

Creo en esta línea que un esfuerzo podría dirigirse a estudiar las interconexiones entre los fenómenos de los diferentes niveles, hoy que ya conocemos y aceptamos la multicausalidad de los fenómenos y con ello la obligación del pensamiento multivariable y no lineal; difícil, porque nuestro modo de pensar tiende a ser univariable, con formulaciones de causalidad muchas veces complejas, pero casi siempre lineales.

Y otro esfuerzo iría a romper la tradición de la oposición entre estructura y proceso o dinámica, que viene marcando una singular oposición, cuando no enfrentamiento, ya histórico y posiblemente inoperante por generador de actitudes excluyentes.

Los pocos psicoterapeutas que en nuestro país (y también fuera) a todo ello dirigen sus esfuerzos y aportaciones son especiales, precisan sólida formación específica, rigurosos conocimientos empíricos, amplia experiencia y cultura, trabajar en clínica fuera de laboratorio, actitudes abiertas, respetuosas y una sensibilidad que les permita alejarse cuando y cuanto sea preciso tanto de las sectas terapéuticas como de lo políticamente correcto. Creo que podemos tener al menos la responsabilidad de prestar nuestro apoyo. Sobre todo cuando también y además se esfuerzan en y para el Sistema Nacional de Salud. 


\section{BIBLIOGRAFÍA}

- Bongar, B. y Beutler L.E. (eds.). Comprehensive textbook of psychotherapy. New York:Oxford University Press. 1995

- Boszormenyi-Nagy, I. Foundation of Contextual Therapy. Brunner/Mazel 1987

- Bowers, K. y Meinchenbaum (eds.). The unconscious reconsidered. New York:Wiley. 1989

- Ceberio, M.R. y Watzlawick, P. La construcción del universo. Conceptos introductorios y reflexiones sobre epistemología, constructivismo y pensamiento sistémico.Barcelona:Herder. 1998

- Fernández Liria, A. y Rodríguez Vega, B. La práctica de la Psicoterapia. Bilbao: Descleé de Brouwer. 2001

- Foerster, H. "Construyendo una realidad". En: P. Watzlawick y otros. La realidad inventada. Barcelona:Gedisa. 1988

- Glasersfeld, E. "Introducción al constructivismo radical”. En: P. Watzlawick y otros. La realidad inventada. Barcelona:Gedisa. 1988

- Guidano, V. The Self in Process:Toward a Post-Rationalist Cognitive Therapy. London:Guilford Press. 1991

- Khun,T. La estructura de las revoluciones científicas. Madrid:Fondo de Cultura Económica. 1975

- Kisch, J. y Kroll, J. Meaning fullness versus Effectiveness: Paradoxical Implications in the Evaluation of Psychotherapy. Psychotherapy: Theory, Research and Practice, 1980. 17, 401-403.

- Lazarus, A.A. The practice of Multimodal Therapy. New York: McGraw-Hill. 1981

- Luborsky, L. y DeRubies R.J. The use of Psychotherapy treatment manuals: A small revolution in psychotherapy research stile. Clinical Psychology Review 1984. 4: 5-14.

- Mahoney, M. y Gabriel, T. Psychotherapy and the cognitive sciences: An evolving alliance. Journal of Cognitive Psychotherapy, 1987. 1, 39-59.

- Maturana, H. "El hombre y la posibilidad del conocimiento objetivo". En: R. Opazo (ed) Integración en Psicoterapia. Santiago de Chile:CECIDEP. 1992

- Morin, E. Ciencia con consciencia. Barcelona:Anthropos. 1984

- Morin, E. "Cultura n Conocimiento". En: P. Watzlawick y P. Krieg (comps.). El ojo del observador. Barcelona: Gedisa. 1994

- Opazo, R. "Fuerzas de cambio en Psicoterapia: Un modelo integrativo". En: R. Opazo (ed.) Integración en Psicoterapia. Santiago de Chile:CECIDEP. 1992

- Packman, M. Construcciones de la experiencia humana (2 vol). Barcelona:Gedisa. 1997

- Pelechano, V. Psicología Sistemática de la Personalidad. Barcelona:Ariel. 2000

- Rojas, E. De la personalidad a la autoestima. Madrid:Temas de Hoy. 2001

- Varela, F. Conocer. Barcelona:Gedisa. 1990

- Watlawick, P. y Krieg, P. El ojo del observador. Barcelona:Gedisa. 1994

- White, M. y Epson, D. Medios narrativos para fines terapéuticos. Barcelona:Paidos. 1993

*Psicóloga Clínica. Psicoterapeuta Sistémica

Servicio de Prevención

Ayuntamiento de Madrid

Fecha de recepción: 16 - XI - 01 